\title{
Positional Communication and Private Information in Honeybee Foraging Models
}

\section{Citation}

Bailis, Peter, Radhika Nagpal, and Justin Werfel. 2010. Positional communication and private information in honeybee foraging models. In Swarm intelligence: 7th international conference, ANTS 2010, Brussels, Belgium, September 8-10, 2010, ed. Marco Doringo. Berlin: Springer. Previously Published in Lecture Notes in Computer Science 6234: 263-274.

\section{Published Version}

doi:10.1007/978-3-642-15461-4_23

\section{Permanent link}

http://nrs.harvard.edu/urn-3:HUL.InstRepos:4728397

\section{Terms of Use}

This article was downloaded from Harvard University's DASH repository, and is made available under the terms and conditions applicable to Open Access Policy Articles, as set forth at http:// nrs.harvard.edu/urn-3:HUL.InstRepos:dash.current.terms-of-use\#OAP

\section{Share Your Story}

The Harvard community has made this article openly available.

Please share how this access benefits you. Submit a story.

\section{Accessibility}




\title{
Positional Communication and Private Information in Honeybee Foraging Models
}

\author{
Peter Bailis ${ }^{1}$, Radhika Nagpal ${ }^{2,3}$, and Justin Werfel $^{3}$ \\ 1 Harvard College, \\ Harvard University, Cambridge, MA, USA \\ pbailis@eecs.harvard.edu \\ 2 School of Engineering and Applied Sciences, \\ Harvard University, Cambridge, MA, USA \\ rad@eecs.harvard.edu \\ 3 Wyss Institute for Biologically Inspired Engineering, \\ Harvard University, Boston, MA, USA \\ justin.werfel@wyss .harvard.edu
}

\begin{abstract}
Honeybees coordinate foraging efforts across vast areas through a complex system of advertising and recruitment. One mechanism for coordination is the waggle dance, a movement pattern which carries positional information about food sources. However, recent evidence suggests that recruited foragers may not use the dance's positional information to the degree that has traditionally been believed. We model bee colony foraging to investigate the value of sharing food source position information in different environments. We find that in several environments, relying solely on private information about previously encountered food sources is more efficient than sharing information. Relying on private information leads to a greater diversity of forage sites and can decrease over-harvesting of sources. This is beneficial in environments with small quantities of nectar per flower, but may be detrimental in nectar-rich environments. Efficiency depends on both the environment and a balance between exploiting high-quality food sources and oversubscribing them.
\end{abstract}

\section{Introduction}

Honeybee colonies are well-known for their ability to coordinate foraging over large areas and efficiently allocate labor among food sources. The predominant model for honeybee communication dictates that bees use a complex movement pattern known as the waggle dance to communicate positional information to unemployed foragers who then proceed to the indicated food source [10], [11]. However, it has recently been suggested that bees may instead rely primarily on private information and use publicly shared information only as a backup [8]. According to recent work, waggle-dancing may act primarily as a trigger that directs bees to forage previously known areas instead of following the dancer's positional cues about a food source. 
We explore the role of private information in bee colony foraging and compare the relative efficiency of foraging in the traditional model of dance communication and in a model in which bees rely solely on their own internal positional information from past experience. We examine each model across several flower densities, distributions, qualities, and nectar quantities in order to determine when communicating positional information is advantageous.

We show that the benefit of sharing position information is highly dependent on the environment in which a colony operates and that relying solely on private information is more efficient than sharing information in several environments. Relying on private information results in a greater number of active forage sites. With small amounts of nectar per flower, this is beneficial as it decreases the risk of quickly exhausting food sources, resulting in fewer wasted foraging trips. Sharing positional information allows the colony to concentrate effort on foraging desirable and energy-efficient food sources at the risk of oversubscribing and quickly depleting them. With nectar-plentiful flowers, the risk of over-harvesting decreases and it becomes beneficial to concentrate foraging efforts.

In Section 2 we discuss related studies and previous models. In Section 3 we present our model and the two communication strategies we consider. Section 4 presents results and analysis. Section 5 concludes.

\section{Related Work}

Colony foraging behavior has been studied extensively, from bee foraging ranges [2] to the distribution of foragers and scouts [10], [16]. A system of advertisement and forager recruitment allows the colony to adjust forager allocation and respond to environmental changes [11]. Bees returning to the hive with food perform a waggle dance, a figure-eight vibration pattern carrying specific positional information [5], the duration of which is proportional to the source's quality [13]. The traditional understanding of the dance is that unemployed bees interpret it as explicit positional directions to a particular forage site [10].

Recent work has suggested that unemployed bees may not make full use of the position information contained in the waggle dance. An alternative hypothesis is that bees use the dance as a cue to return to previously discovered, privately known food sources [3], [8]. In one study, 93\% of waggle dance recruits returned to internally remembered food sources rather than the source designated by dancers [7]. These findings are opposed to the traditional understanding of bee behavior, yet the extent to which bees rely on internal information and the relative benefits of sharing information are still in question [4], [14].

Prior work has studied the efficiency of the waggle dance in particular environments and communication models, but has not considered the potential role of private bee memory. Dornhaus et al. considered the role of recruitment and colony size within simulation and found that communication conferred the greatest benefit in worlds with few food sources arranged in scarce patches [6]. Beekman and Lew further explored the role of communication and found that communication allows colonies to efficiently exploit the most profitable food sources. 
The authors considered several communication models, including a model where recruits searched for new food sources instead of following dance information [1], but recruits did not use any private information about previously known food sources. In our work, we focus on the recent discussions regarding the role of private information in bee forager allocation.

\section{Model of Colony Foraging}

We constructed an agent-based model that reflects current knowledge of bee foraging in nature.

\section{$3.1 \quad$ World}

We model a continuous world with discrete timesteps. The world is $12 \mathrm{~km}$ by $12 \mathrm{~km}$ with a hive at the center, corresponding to a $6 \mathrm{~km}$ radius of foraging activity [2], and bees cannot travel beyond its boundaries. Each timestep in our world corresponds to approximately 3.5 seconds of real time.

\subsection{Flowers}

Flowers represent food sources, and each has a predetermined quantity of nectar that can support a fixed number of forager trips before being exhausted. Nectar qualities are variable: flowers can contain either low-quality nectar (1 unit per trip) or high-quality nectar (4 units per trip), reflecting the proportions of sugar concentrations found in different flowers [6]. Because we model a short timeframe on the order of days, we do not simulate flower death.

We model two possibilities for flower distribution: evenly scattered and clustered (Fig. 1). In scattered worlds, food sources are randomly placed, similar to Dornhaus et al. [6]. In clustered worlds, food sources are distributed among a number of randomly placed clusters, and each flower is placed at a distance normally distributed ( $\mu=0, \sigma=72$ meters) from the cluster center, similar to Beekman and Lew [1]. Each cluster is of one particular quality, and we probabilistically add additional clusters at a predetermined rate in order to simulate a dynamic environment. We begin with six clusters in our world and end with approximately 10.5 clusters at the end of 100 hours.

Given a flower $f$ distance $D_{f}$ from the hive with nectar quality $N_{f}$ and $D_{\max }$, the maximum distance from the hive, we define the overall flower quality $Q(f)$ :

$$
Q(f)=\frac{D_{\max }-D_{f}}{D_{\max }} * N_{f}
$$

This equation closely resembles a proposed metric for assessing flower desirability, (expected energy obtained - cost of trip)/(time of trip) [12], and prioritizes high-quality flowers closer to the hive, optimizing trip energy cost compared to the expected nectar yield. Under this model, a low-quality flower at distance $n$ will be valued equivalently to a high-quality flower at distance $4 n$. 

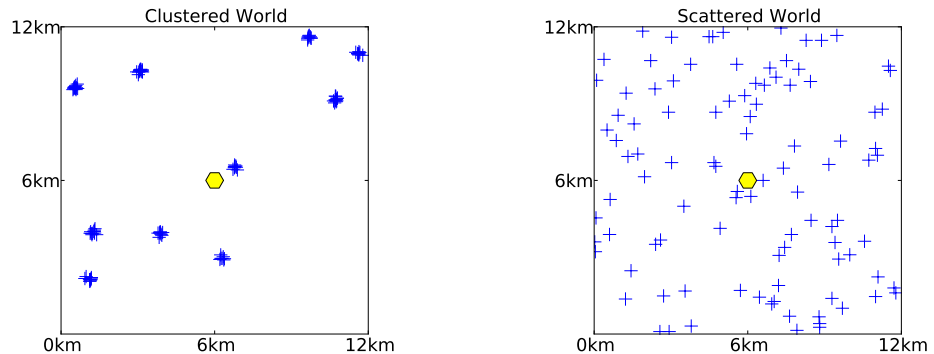

Fig. 1. Representative food distributions. Each world contains 100 flowers $(+)$ and the hive (hexagon). Flowers are not drawn to scale.

\subsection{Bees and Movement}

We model foraging bees as simple agents. Bees can fly at a speed of $25 \mathrm{~km} / \mathrm{h}$ in any direction and can detect and harvest nectar from flowers within a radius of $24 \mathrm{~m}$ [6]. Bees remember one flower location at a time and harvest nectar from one flower per trip from the hive. A bee can travel up to $6 \mathrm{~km}$ before it must return to the hive to replenish its energy stores. In the hive, bees deposit collected nectar.

We model the inaccuracies of bee flight directly instead of artificially causing flower location efforts to fail as in prior work [1] using two mechanisms: actuation error and perception error. At each timestep, the bee calculates its desired trajectory based on a goal position and the position at which it currently believes it is located. Because bee flight is imperfect, its actual trajectory is the desired trajectory plus a small perturbation, the actuation error. Sensory feedback lets the bee update its estimate of its position based on the actual rather than desired trajectory. However, sensing is imperfect, so the bee's position estimate is updated by the actual trajectory plus another perturbation, the perception error. For our simulations, we used independent perception and actuation errors randomly chosen between $-3 \%$ and $3 \%$ per bee per timestep which were applied to the magnitude and angle of the movement vectors. To model familiarity with areas closer to the hive, when returning bees are within a short distance from the hive $(120 \mathrm{~m})$, their perceived position is updated to accurately reflect their actual position.

\subsection{Bee roles}

Bees have one of three roles: scout, forager, or unemployed, similar to roles considered in related work [1], [6], [15].

A scout searches for new flowers by flying away from the hive and moving randomly throughout the world according to a Lévy flight pattern, a random walk with step length $l$ distributed according to an inverse power law $P(l) \propto l^{-2}$ such that $l \in[24 \mathrm{~m}, 6 \mathrm{~km}]$ that is believed to closely approximate bee flight 

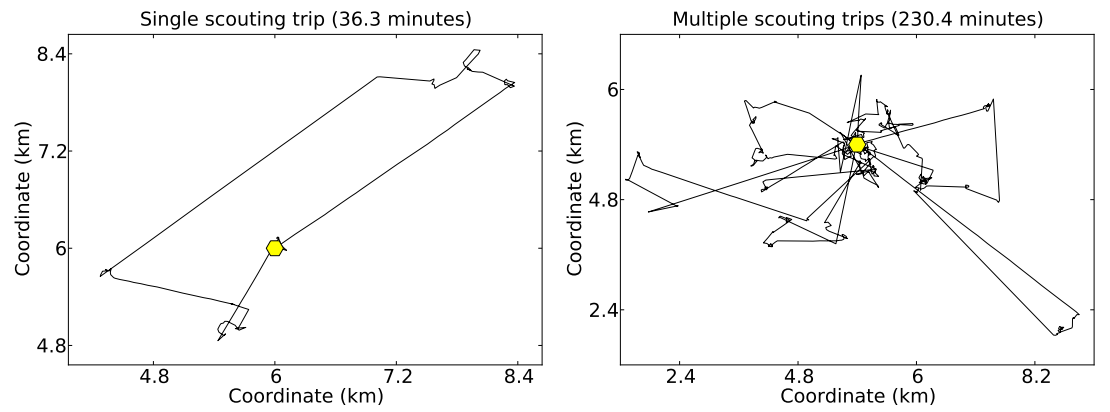

Fig. 2. Bee movement (Lévy flight) in single and multiple scouting trips from the hive.

in nature [9] (Fig. 2). At each timestep, a scout surveys its surroundings and remembers the highest quality flower it has observed according to Equation 1. Upon depleting its energy, the scout returns to the hive, where it replenishes its energy supply and becomes a forager.

A forager is aware of a flower's position and repeatedly flies to it, collects nectar, and delivers the nectar to the hive. Because a bee's perceived position is not necessarily the same as its actual position, the coordinates it remembers may not reflect the flower's actual position in the world. When a forager perceives it has reached its remembered coordinates, it attempts to locate and harvest a flower. If none are in the vicinity whether due to positional error or flower depletion, the forager begins Lévy flight and searches for a new flower which it will then collect nectar from and subsequently remember according to its perceived position. Once the forager collects nectar or runs out of energy, it must return to the hive. Therefore, foragers who reach their known flower position but do not find a flower become similar to scouts, but upon finding nectar they collect it and return (Fig. 3).

An unemployed forager has no known flower and must either wait to be recruited or become a scout. At each timestep, with a small probability (.1\%), it becomes a scout and searches for a food source [3], [15].

\subsection{Flower Quality, Foraging, and Recruitment}

Upon returning to the hive with a known flower location, a bee has three choices: forget about the known flower, continue to forage from the flower, or continue to forage from the flower after attempting to recruit additional foragers.

In order to adapt to changing environments and different conditions, bees maintain dynamic thresholds that determine whether a bee should remember or forget about a current food source as well as whether a bee should advertise the flower using the waggle dance [11], [12]. While the threshold for abandoning a source is a function of the individual flower, the threshold for dancing is complicated and depends on colony-wide metrics like the ability to find a 

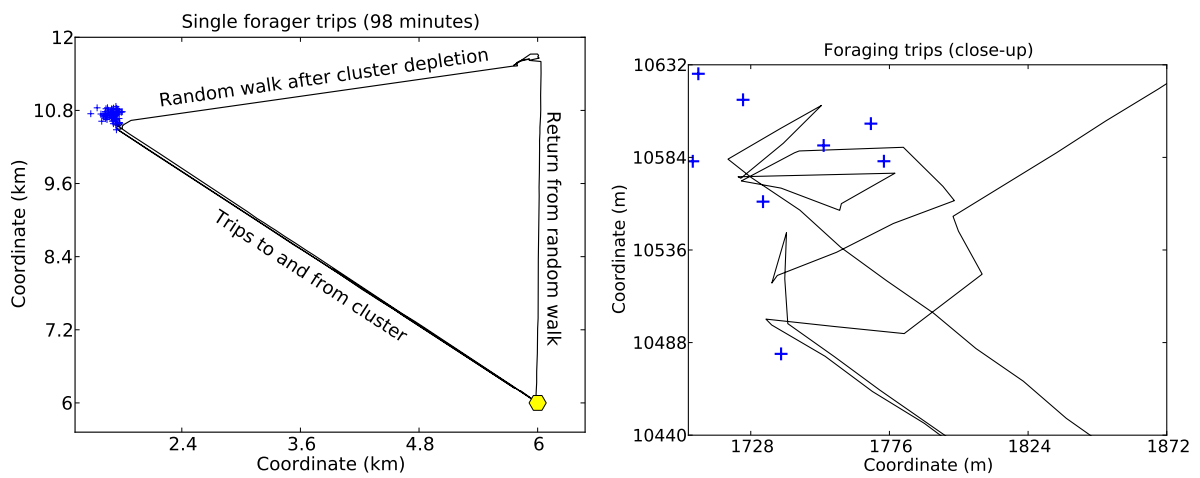

Fig. 3. Movement of a single bee making foraging trips to and from a cluster of flowers. After one successful trip, the bee returns to the patch and, because the nearby flowers are depleted (due to other bees foraging as well), the forager begins Lévy flight in search of a new flower (traveling to the right corner), eventually returning to the hive. Note that even though the forager believes it has returned to the same position each trip, the actual positions at which it arrives are slightly different due to movement error, as seen in the close-up.

food-storer bee [15]; because we do not model these features of the colony, we use individually-tuned threshold mechanisms for both which quickly converge at the colony level in practice. Each bee has a harvest threshold, $t_{h}$, and a dance threshold, $t_{d}$, with the invariant that $t_{h} \leq t_{d}$. Each time a bee returns to the hive with a known flower, it assesses the quality relative to its thresholds in order to determine the appropriate action. The chosen action also affects the thresholds, creating a dynamic feedback mechanism.

Given a flower $f$ with quality $q=Q(f)$, we have three possibilities:

$q<t_{h}$ : The flower quality is poor relative to the flowers the bee has recently encountered. The bee becomes unemployed, but lowers its standards for foraging:

$$
t_{h} \leftarrow t_{h}-\frac{t_{h}-q}{2}
$$

$t_{h}<q<t_{d}$ : The flower quality is better than others that the bee has recently encountered, but not of sufficient quality to merit advertising to other bees. The bee remembers the flower and continues to harvest it. The bee raises its standards for harvesting and lowers its standards for advertising.

$$
t_{d} \leftarrow t_{d}-\max \left(.1, \frac{t_{d}-q}{2}\right), t_{h} \leftarrow \min \left(t_{h}+\max \left(.1, \frac{q-t_{h}}{2}\right), t_{d}\right)
$$

$q>t_{d}$ : The flower quality is higher than the flowers that the bee has recently encountered. The bee remembers the flower, advertises it to other bees, and continues to harvest it. The bee raises its standards for advertising: 


$$
t_{d} \leftarrow t_{d}+\frac{q-t_{d}}{2}
$$

In order to model a scout's willingness to find new food sources, scouts halve both of their thresholds upon leaving the hive.

\subsection{Hypothetical Communication Models}

Bees advertise flowers by "dancing" inside the colony. Given a flower $f$ of quality $q=Q(f)$, if a bee decides to advertise $f$, it will do so for $c * q$ timesteps. In our model, $c=5$. A bee can recruit exactly one other bee per timestep as only a limited number of bees can physically observe a waggle dance at a given time. Recruits are chosen randomly from the pool of unemployed bees [12].

We consider two possible communication models for recruitment:

- Model SharePosition. In this traditional position-sharing model, recruits become active foragers at the advertised position. Because the flower coordinates are specified according to the dancer's perceived location from when it found the flower, the recruit may not find it due to actuation and perception errors of both the dancer and the recruit or because of flower depletion.

- Model PrivatePosition. In this private-information model, recruits ignore the positional information a dancer provides and begin to forage at their previously known flower position, or, if they have none, they become scouts. This model deemphasizes the role of positional information in foraging coordination and instead focuses on private information [8]. In this model, dancing reactivates unemployed foragers instead of providing direction regarding where to forage.

These two models represent the accepted understanding of bee communication as well as new theories about bee communication, respectively.

\section{Results}

We evaluated the relative efficiency of the communication models in several distinct environments within a custom simulator, varying flower generation rates, flower distributions, and flower nectar quantities (Fig. 4). For each configuration, we simulated 96 hours of foraging with a colony of 500 bees. We do not consider different colony sizes, but consistent with prior work [6], colony size did not significantly impact our results.

Relative foraging efficiency (nectar gathered per unit of energy expended) depended greatly on the environment; in several cases PrivatePosition outperformed SharePosition (up to 40\%), but, in others, SharePosition was more efficient (up to 35\%). At low flower generation rates, PrivatePosition performed better in scattered worlds than in clustered worlds, but, at high flower generation rates, it performed better in clustered worlds. PrivatePosition's relative 

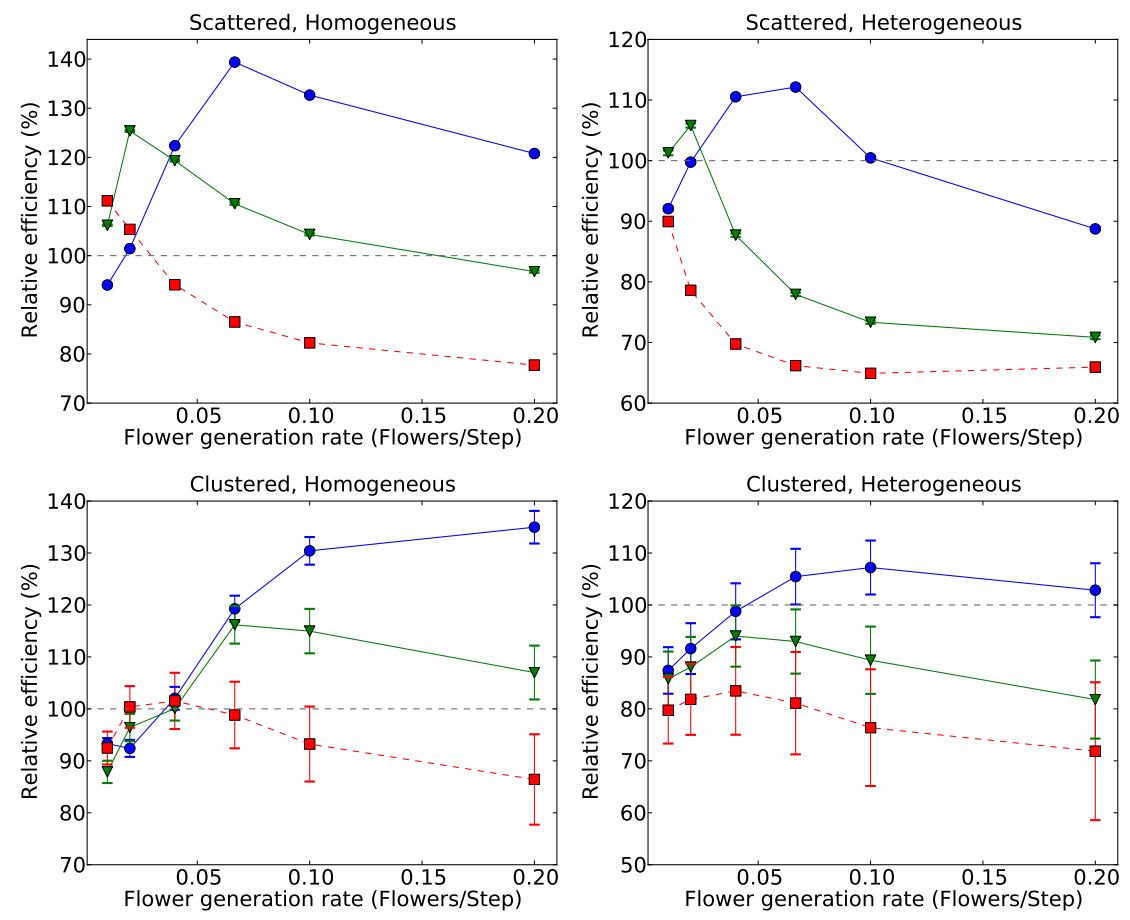

$\because 20$ Trips per Flower

$\square 200$ Trips per Flower

Fig. 4. Relative efficiency of PrivatePosition compared to SharePosition. Efficiency is defined as the ratio of nectar retrieved to energy expended. Each data point represents 100 trials in separate, randomly-generated environments matching the specified configuration. Error bars show the standard error on the mean.

performance was better in homogeneous worlds than in heterogeneous worlds. In general, as nectar quantity increased such that a given flower could support more repeated trips, the relative efficiency of PrivatePosition decreased. Similarly, as flower generation rates increased, PrivatePosition became less effective.

The food distribution influenced the usefulness of information sharing. In scattered worlds, bees were likely to find flowers independently, especially at moderate to high flower generation rates; sharing did not confer a serious advantage because flower locations were not geographically correlated. In clustered worlds, however, once a bee found a cluster, it effectively found many flowers, and sharing information allowed bees to find clumps faster than if they searched independently. With heterogeneous nectar qualities, however, sharing information about food sources became more valuable as coordination allowed the colony to concentrate on both nearby flowers and higher quality nectar. 


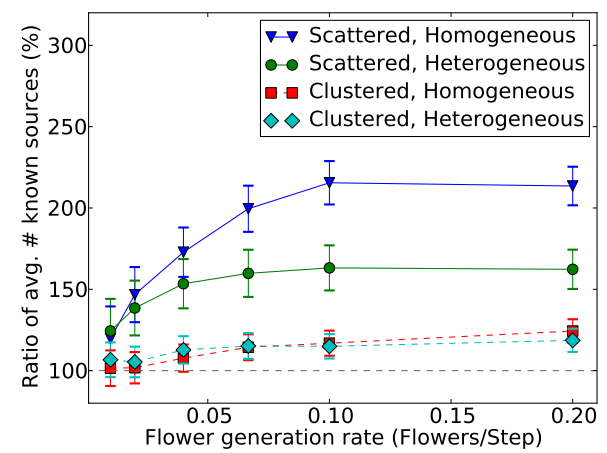

Fig. 5. Average number of clusters or flowers known to actively foraging bees at each timestep, expressed as a ratio of PrivatePosition to SharePosition with flower nectar quantity limited to 20 trips. If a bee knew of a food source, had visited it, and the source was not depleted, then the colony was considered aware of the food source. The clustered worlds contained 10 randomly placed flower clusters and we counted a flower as belonging to a cluster if the flower was within $480 \mathrm{~m}$ of the cluster center. If a flower was located within multiple cluster radii, we counted the flower as belonging to each cluster. Each data point represents the average of 30 trials, and error bars show the standard error on the mean.

PrivatePosition was aware of a greater number of food sources on average than SharePosition, which had several consequences for efficiency. We examined the average number of food sources the colony exploited at any given time (Fig. 5), and found that PrivatePosition foraged up to $114 \%$ more flowers in scattered worlds and $25 \%$ more clusters in clustered worlds. As the number of known food sources decreased, each was visited by a greater number of foragers and was depleted more quickly. Once depletion occurred, foragers needed to find a replacement flower, which, depending on the environment, could be an energyintensive process. In scattered worlds, adding additional flowers made it easier to find a replacement. In clustered worlds, however, adding more flowers only increased the amount of food at existing cluster locations and did not contribute additional geographic diversity to the food distribution. This partly explains the noise in the cluster scenarios: placing flowers at a cluster had similar effects to placing a single large, high-capacity flower. As long as some flowers remained in a cluster, foragers could likely find a replacement, but, if a cluster was entirely depleted, finding another food source nearby was unlikely. Because flowers were constantly generated, subsequent returns to a depleted cluster would possibly yield some nectar, which is advantageous for PrivatePosition, however at low rates of generation this resulted in many wasted trips.

SharePosition favored foraging from better food sources and quickly depleting them, while PrivatePosition favored foraging from many possibly inferior sources and had more successful forager trips. We examined the number of foraging trips that did not result in nectar harvesting (Fig. 6), and SharePosition consistently 

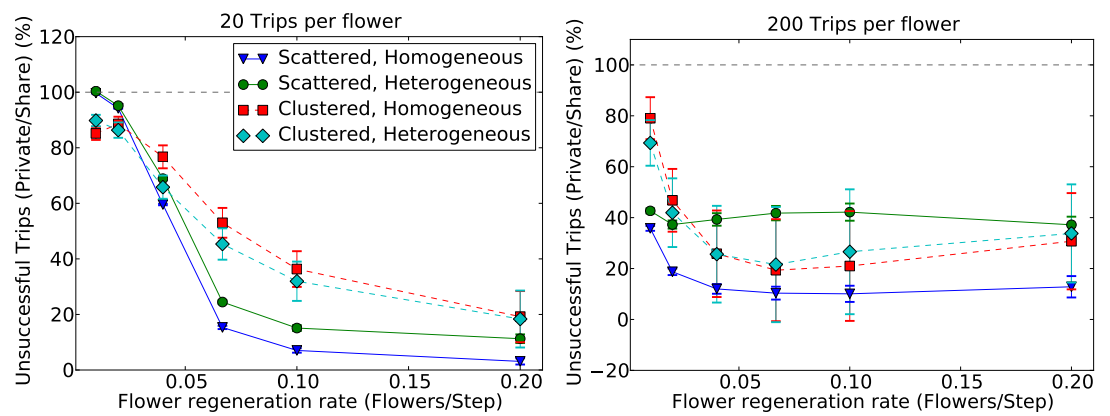

Fig. 6. Unsuccessful foraging trips as a fraction of the total number of foraging trips in PrivatePosition compared to SharePosition across multiple environments. A trip was unsuccessful if a forager returns to the hive without food. Each data point represents 100 different simulated worlds, and error bars show the standard error on the mean.

had a higher proportion of unsuccessful trips. SharePosition was more effective with high flower nectar quantities than with low flower nectar quantities, but was still less successful overall than PrivatePosition. In worlds with few flowers, the two models were almost equivalent because food sources were hard to find and easy to deplete. Even though recruits in SharePosition received position information, they were less successful than in PrivatePosition; SharePosition foragers did not find their food sources as often as PrivatePosition foragers did.

PrivatePosition's relative foraging success was due to SharePosition's oversubscription of the more favorable food sources. At low flower nectar quantities, SharePosition's concentration of foraging efforts resulted in rapid source depletion, wasted trips, and difficulty finding new flowers. In scattered, homogeneous worlds, for example, in PrivatePosition, the colony harvested from a wide range of food sources, while in SharePosition, the colony harvested from food sources only within a close radius of the hive, decreasing flight time but increasing its proportion of unsuccessful trips. Increasing flower nectar quantity reduced the risk of oversubscribing any particular food source and supported greater forager concentration. With higher nectar quantities, SharePosition still had fewer successful trips, but each successful trip was more valuable (due to trip speed or nectar quality), resulting in a higher overall efficiency (Fig. 4). Similarly, with one trip-worth of nectar per flower, there would be no benefit to sharing information (except due to geographic flower locality), but with infinite nectar per flower, sharing information would greatly benefit the colony.

The colony must balance the tradeoff between focusing on fewer, more desirable food sources and oversubscribing them. While sharing information about flowers in SharePosition resulted in higher nectar quantities, if a food source was quickly depleted by foragers, the benefit of sharing information decreased due to wasted energy and forager effort. Efficiency largely depended on whether, by recruiting additional foragers to a food source, the colony wasted energy upon 
source depletion that could have been avoided by spreading out the foragers among other sources instead. As flowers supported more foragers, the colony could concentrate on better food sources without the risk of quick depletion, and the benefit of sharing information increased.

We summarize several of the important factors in determining model success:

- When the nectar quantity per food source increases, the importance of harvesting from a variety of food sources decreases as depletion slows. The benefit of sharing information about high-quality food sources increases.

- Exploiting a variety of food sources is useful when they are easily depleted, but, as food sources become easier to find, oversubscribing is less harmful as foragers are more likely to find replacement flowers.

- In heterogeneous environments, exploiting the most profitable food sources may outweigh the cost of over-harvesting, so position sharing is more beneficial. In homogeneous worlds, this effect is lessened as the difference between the best and worst sources is smaller.

- In environments with many flowers located closely together (as in clustered worlds), the importance of exploiting a variety of food sources decreases compared to environments with scattered food sources; it is easier to find another flower nearby once a targeted flower becomes depleted. However, once a cluster is depleted, it may be difficult to find a new food source.

\section{Conclusion}

In evaluating the benefit of communicating positional information in bee foraging, one must consider a variety of factors: flower quality, quantity, capacity, and distribution. Our results show that, under appropriate conditions, relying on internal information can be more efficient than sharing information. This supports recent studies about private information that run contrary to the traditional interpretation of information sharing in honeybees. It is plausible that this behavior occurs naturally as a response to particular environment types, particularly when food source diversity is important.

In this work, we have provided evidence for the efficiency of relying on private information within a bee foraging model in several environments and explained factors for each model's success. Our results have consequences not only for biologists but for system designers who are faced with decisions about communication and task allocation in a particular environment. Depending on environmental characteristics, the additional costs of incorporating a communication system may outweigh the benefit of doing so if one can instead rely on internal agent memory. While field biologists have not yet conclusively determined the importance of sharing positional information in honeybee foraging, we have shown that in some environments relying solely on private information may be more efficient than sharing position information.

Source code and Traces. All source code and traces are available at http://eecs.harvard.edu/ pbailis/beesim/. 
Acknowledgements. The authors would like to thank Peter Lifland for his assistance with early simulation and modeling. This work was supported in part by NSF Expeditions Grant IIS-926148 and the Wyss Institute for Biologically Inspired Engineering.

\section{References}

1. Beekman, M., Lew, J.B.: Foraging in honeybees-when does it pay to dance? Behavioral Ecology 19(2), 255-261 (2008)

2. Beekman, M., Ratnieks, F.L.W.: Long-range foraging by the honey-bee, Apis mellifera L. Functional Ecology 14, 490-496 (2000)

3. Biesmeijer, J.C., Seeley, T.: The use of waggle dance information by honey bees throughout their foraging careers. Behavioral Ecology and Sociobiology 59, 133$142(2005)$

4. Brockmann, A., Sen Sarma, M.: Honeybee dance language: is it overrated? Trends in Ecology and Evolution 24, 583 (2009)

5. Dornhaus, A., Chittka, L.: Why do honey bees dance? Behavioral Ecology and Sociobiology 55, 395-401 (2004)

6. Dornhaus, A., Klügl, F., Oechslein, C., Puppe, F., Chittka, L.: Benefits of recruitment in honey bees: effects of ecology and colony size in an individual-based model. Behavioral Ecology 17(3), 336-344 (2006)

7. Grüter, C., Balbuena, M., Farina, M.: Information conflicts created by the waggle dance. Proceedings of the Royal Society Biological Sciences 275, 1327 (2008)

8. Grüter, C., Farina, W.: The honeybee waggle dance: can we follow the steps? Trends in Ecology and Evolution 24, 242-247 (2009)

9. Reynolds, A.: Cooperative random lévy flight searches and the flight patterns of honeybees. Physics letters A 354, 384-388 (2006)

10. Seeley, T.: Division of labor between scouts and recruits in honeybee foraging. Behavioral Ecology and Sociobiology 12, 253-259 (1983)

11. Seeley, T.: Honey bee foragers as sensory units of their colonies. Behavioral Ecology and Sociobiology 34, 51-62 (1994)

12. Seeley, T.: The Wisdom of the Hive: The Social Physiology of Honey Bee Colonies. Harvard University Press, Cambridge, MA (1996)

13. Seeley, T., Mikheyev, A.: Dancing bees tune both duration and rate of wagglerun production in relation to nectar-source profitability. Journal of Comparative Physiology A 186, 813-819 (2000)

14. Shermin, G., Visscher, P.: Honeybee colonies achieve fitness through dancing. Nature 419, 920-922 (2002)

15. de Vries, H., Biesmeijer, J.C.: Modelling collective foraging by means of individual behaviour rules in honey-bees. Behavioral Ecology and Sociobiology 44, 109-124 (1998)

16. Waddington, K., Holden, L.: Optimal foraging: on flower selection by bees. The American Naturalist 114 (1979) 